\title{
EQUITABLE SELECTION IN BILATERAL MATCHING MARKETS
}

\begin{abstract}
This paper presents a procedure to select equitable stable allocations in two-sided matching markets without side payments. The Equitable set is computed using the Equitable algorithm. The algorithm limits the set of options available for each agent throughout the procedure. The stable matchings selected are generally not extreme, form a lattice and satisfy the condition of being "Ralwsian" in each partition of the market. The Equitable algorithm can also be used to select a particular matching from the Equitable Set favoring particular agents independent of the side of the market to which they belong.
\end{abstract}

KEY WORDS: matching markets, fair distribution, fair algorithm.

\section{INTRODUCTION}

Consider the following situation: Imagine we have to form groups of surgeons and anesthetists. Surgeons will have different levels of expertise and fields, and the anesthetist also will have different levels of expertise. Both groups have preferences over the members of the other groups. To allocate them we can use different procedures preserving the stability of the final outcome. If we decide to match them using the deferred acceptance procedure, we shall ask them for their preferences on their potential matches, and decide whether to use the algorithm where surgeons propose first, or the other way around. The group proposing will be favored in the final matching. However, both groups are experts in their fields and there is no trivial reason to impose one group's preferences over the other. There might even be some evidence that balanced teams, in which none of the agents has his best partner but none has his worst either, perform better. Therefore, we need an algorithm able to compromise between both groups' ideal match. 
The need for compromise solutions is particularly acute in bilateral matching markets. In these markets, the set of stable solutions reflects the strong polarization of the interest that the participants have, i.e., the best stable matching for surgeons is the worst possible matching for anesthetists and vice versa.

This paper proposes a way to select compromise stable matchings in one-to-one matching markets without side payments. The selected stable matchings are called Equitable. This set of solutions is fair, fairness being defined in the sense of Rawlsian justice (Rawls, 1971). It also selects a subset of stable matchings and gives the planner control over the final outcome.

The literature on matching markers presents different approaches to compromise solutions, which are in some sense fair. An axiomatic approach to the problem of fair algorithms was presented in Masarani and Gokturk (1989). They define a fair matching algorithm by a set of four axioms (Gender Indifference, Peer Indifference, Maximin Optimality, and Stability). They show that not all preference profiles admit a fair matching algorithm, and that even the algorithm generating the mutual agreement match has no chance to be fair. This approach concentrates on the algorithm, not on the resulting matchings.

In a transferable utility context, Bennett (1988) characterizes bargaining consistent conjectures and the (stable) matchings that are stable with respect to these conjectures. Assuming a particular bargaining structure, Rochford (1984) develops the set of Symmetrically Pairwise-Bargained allocations. Roth and Sotomayor (1998) have shown how this set reflects the same polarization of interest that characterizes the core of the matching markets. These results suggest some avenues through which to address the issues of equity and distribution that arise in two-sided matching markets. At the same time, they show that the polarization of interests that exists in the core of two-sided matching markets may be even more fundamental than previous results have suggested.

In models where participants' preferences are assumed to be ordinal, most solution criteria impose cardinality over participants' preferences. The first of these results was attributed to Selkow by Knuth (1976) and it is inspired by the utilitarian 
solution. Another approach in the same vein is the Sex-Equal Matching (SEM) presented by Romero-Medina (2001). This measure is based on the minimization of the envy difference between the sets of men and women, while preserving stability. The imposition of cardinality implies problems in the measurement of the intensity of the participants' preferences. Furthermore, the stable matchings that are selected are usually not unique and have no particular structure.

This paper maintains the ordinal framework and presents results similar to those produced by a bargaining process within the context of transferable utility. The paper's results are obtained by fixing an arbitrary order of the participants and limiting the set of acceptable partners for the participants in the market throughout the procedure. These two characteristics impose a power balance between the participants involved in the allocation process. This fact characteristic trickles down to in the stable matchings selected by the procedure. The set of Equitable matchings is not empty and generally different from the men's or women's optimal stable matching for a particular market. The matchings in the Equitable Set form a lattice and satisfy the condition of being Rawlsian in each partition of the market. The matching selected by the procedure depends on the order in which the participants make proposals on the procedure. The paper shows how this order can be used to give the planner some control over the final allocation.

The rest of the paper is organized as follows: Section 2, provides a formal definition of concepts. the Equitable Algorithm is defined in Section 3. In Section 4, the main results are presented. Section 5 analyzes the concept of "Rawlsian by partitions" that is satisfied by the set of Equitable matchings. The last section is devoted to some final remarks.

\section{THE MODEL, NOTATION, AND DEFINITIONS}

Let us consider a bilateral market with two finite disjoint sets $M=\left\{m_{1}, m_{2}, \ldots, m_{n}\right\}$ and $W=\left\{w_{1}, w_{2}, \ldots, w_{m}\right\}$, the sets of men 
and women, respectively. Here $m_{i}$ 's preferences $P_{m_{i}}$ are described by a linear order on $W \cup\left\{m_{i}\right\}$. Given to women $w_{j}, w_{h} \in W$, the expression $w_{j} P_{m_{i}} w_{h}$ means that $m_{i}$ prefers to be matched to $w_{j}$ rather than $w_{h} ; m_{i} P_{m_{i}} w_{h}$ means that $m_{i}$ prefers to stay single rather than been matched to $w_{h}$. Similarly, each woman $w_{j}$ 's preferences $P_{w_{j}}$ are described by a linear order on $M \cup\left\{w_{j}\right\}$. The preferences of any participant are represented by a list, $P_{m_{i}}=$ $w_{1}, w_{2}, \ldots, m_{i}, w_{t}, w_{t+1}, \ldots$, where $w_{1}$ is $m_{i}$ 's most preferred mate, $w_{2}$ is his second most preferred mate and so on. Throughout the paper, it is assumed that no participant is indifferent between any two potential mates, or between any possible mate and the nomarriage option. The marriage problem is fully described by a triplet $(M, W, P)$, where $P$ is a preference profile containing a full description of the agent's preferences.

A matching is a function $\mu: M \cup W \rightarrow M \cup W$, such that

(1) $\left(\mu\left(m_{i}\right) \notin W \Rightarrow \mu\left(m_{i}\right)=m_{i}\right)$, and $\left(\mu\left(w_{j}\right) \notin M \Rightarrow \mu\left(w_{j}\right)=w_{j}\right)$, and

(2) $\mu\left(m_{i}\right)=w_{j} \Leftrightarrow \mu\left(w_{j}\right)=m_{i}$.

A matching $\mu$ is blocked by an individual $m_{i}$ in the market $(M, W, P)$ if and only if $m_{i} P_{m_{i}} \mu\left(m_{i}\right)$. A matching that cannot be blocked by any individual is called individually rational. A matching $\mu$ is blocked by a pair $\left(m_{i}, w_{j}\right)$ in the market $(M, W, P)$ if and only if $w_{j} P_{m_{i}} \mu\left(m_{i}\right)$ and $m_{i} P_{w_{j}} \mu\left(w_{j}\right)$. Any individually rational matching for $(M, W, P)$ that cannot be blocked by pairs is said to be stable for this market. In this matching market the set of stable matchings coincides with the core of this market.

Let $\Gamma(P)$ be the set of all possible stable matchings in the market $(M, W, P)$. The set of stable matchings forms a distributive lattice under a natural ordering relation. It implies the existence of $M$-optimal and $W$-optimal stable matchings. Let us denote these matchings by $\mu^{M}$ and $\mu^{W}$, respectively. The $\mu^{W}$ stable matching is the worse stable matching for all the men and vice versa. 


\section{THE EQUITABLE ALGORITHM}

In this section, the Equitable Algorithm is introduced. The main characteristic of the Equitable Algorithm is that it requires "patient" behavior on the participants. This behavior is modeled so as to not allow the participants to accept proposals that can damage them severely in the first stages of the procedure.

The Equitable Algorithm requires the order of the participants to be fixed. This order plays a relevant role in the final output of the process. Let $\Phi^{*}$ be the equitable algorithm with $\zeta^{*}$ as the starting order for the participants. Let $\mu_{\Omega}^{*}$ be the outcome of $\Phi^{*}$.

\section{EQUITABLE ALGORITHM $\Phi^{*}$ :}

- Step 0: We fix an arbitrary order $\zeta^{*}$ for the participants.

- Step 1: The first agent in $\zeta^{*}$, say $m_{1} \in M$, makes an offer to the first women on his preference list of acceptable women. The women who receives the proposal accepts $m_{1}$ if he is her first option in her preference list. Otherwise she rejects $m_{1}$. If she accepts the offer of $m_{1}$ she blocks man $m_{1}$ to make any further offer. The algorithm proceeds in the same way, following the order in $\zeta^{*}$, for all the participants in $\zeta^{*}$ that are not blocking an offer.

- Step $k$ : the first person in $\zeta^{*}$ among the men and women that are not blocked or are not blocking an offer after Step $k-$ 1 , are called $p \in M \cup W$. They propose to his/her most preferred person on its preference list of acceptable participants. The person who receives the proposal accepts it if $p$ is among her/his $k$ best choices and/or is better than the proposal she/he is blocking, otherwise she/he rejects $p$. In case of a rejection $p$ proposes to his/her second most preferred choice among his/her $k$ first choices. The algorithm proceeds in the same way, following the order in $\zeta^{*}$, for all the participants in $\zeta^{*}$ that are not blocking an offer, i.e., are available.

If a proposal is accepted by an agent that is blocking an offer, the next agent to propose will be the one that was held by him/her. After this, the algorithm proceeds 
following the order in $\zeta^{*}$, for all the participants in $\zeta^{*}$ that are not blocking an offer.

- Stop: The Algorithm terminates at a step where for each men and women the following holds: either $p$ 's proposal is held by some other person in the other side of the market, or $p$ has proposed to all of his/her acceptable partners. The outcome of the algorithm is the matching at which each person is matched to the person that he or she is blocking when the algorithm stop. Persons who did not receive a proposal or are rejected by all the persons he/she proposes to, remain unmatched. We denote by $\Phi^{*}[P]$ the matching that algorithm $\Phi^{*}$ yields when applied to a profile $P$.

PROPOSITION 1. The Equitable Algorithm $\Phi^{*}$ always generates a stable matching according to $P$.

Proof. We first check that the outcome of $\Phi^{*}$ is stable. The proof proceeds by contradiction. Suppose that at the end of the procedure we can find a blocking pair $\left(m_{b}, w_{b}\right)$ in the resulting allocation. It means that there exists a blocking pair such that $w_{b} P_{m_{b}} \mu_{\Omega}^{*}\left(m_{b}\right)$, and $m_{b} P_{w_{b}} \mu_{\Omega}^{*}\left(w_{b}\right)$.

Given that the algorithm has terminated, then either both agents are blocking an offer or at least one of them is unmatched.

Assume, w.l.o.g., that $m_{b}$ is unmatched at the end of the procedure. If at least one agent in a blocking pair remains unmatched on $\mu_{\Omega}^{*}$ and the algorithm $\Phi^{*}$ has ended, it means that the unmatched agents has proposed in the last round of the algorithm to all his acceptable partners including $w_{b}$, and $w_{b}$ rejects his offer. In this case $\mu_{\Omega}^{*}\left(w_{b}\right) P_{w_{b}} m_{b}$ and therefore $\left(m_{b}, w_{b}\right)$ is not a blocking pair. The same argument holds if both agents are unmatched at the end of the procedure.

Assume that both agents are blocking an offer at the end of the procedure such that $\mu_{\Omega}^{*}\left(m_{b}\right) \neq m_{b}$ and $\mu_{\Omega}^{*}\left(w_{b}\right) \neq w_{b}$, and the pair $\left(m_{b}, w_{b}\right)$ blocks $\mu_{\Omega}^{*}$. If this is the case, it must be that both agents have received early proposals that they accept without having the chance to interchange proposals. Assume that $w_{b}$ was blocking a proposal from $\mu_{\Omega}^{*}\left(w_{b}\right)$ before 
she becomes acceptable for $m_{b}$ and $m_{b} P_{w_{b}} \mu_{\Omega}^{*}\left(w_{b}\right)$. In that case, we shall prove that it is impossible for $m_{b}$ to hold a proposal $\mu_{\Omega}^{*}\left(m_{b}\right)$ such that $w_{b} P_{m_{b}} \mu_{\Omega}^{*}\left(m_{b}\right)$ before proposing to $w_{b}$.

Suppose that $m_{b}$ accepts a proposal before $w_{b}$ is acceptable for him and blocks it until the algorithm has ended. In that case $\mu_{\Omega}^{*}\left(m_{b}\right) P_{m_{b}} w_{b}$, a contradiction.

Suppose that $m_{b}$ accepts a proposal before $w_{b}$ is acceptable for him and blocks it until $w_{b}$ accepts a proposal and this proposal is dropped by $\mu_{\Omega}^{*}\left(m_{b}\right)$ before the algorithm finishes. In this case, the procedure gives the chance to $m_{b}$ to propose to all his acceptable partners before any acceptable partner can hold him. In that case if $m_{b} P_{w_{b}} \mu_{\Omega}^{*}\left(w_{b}\right)$ then $w_{b}$ can drop her present match and accept $m_{b}$ 's proposal. If $m_{b}$ does not offer to $w_{b}$ it is because

- she is not acceptable to him yet or,

- because an offer for a better match is accepted first.

If $w_{b}$ is not acceptable at this stage and $m_{b}$ becomes held before she is acceptable then $m_{b}$ is held by an agent he prefers over $w_{b}$. If not, then $w_{b}$ is acceptable and $m_{b}$ will propose to her. If she rejects it is because she is blocking a better offer. Therefore $\left(m_{b}, w_{b}\right)$ is not a blocking pair at this stage.

If along the algorithm $w_{b}$ lost her tentative partner she will propose immediately after and the same argument follows. Therefore the algorithm will not permit the existence of blocking pairs.

Let us fix an arbitrary order $\zeta^{M}$ for the participants under the restriction that all men precede all women. Let $\Phi^{M}$ denote the Equitable Optimal Algorithm for men, i.e., when $\zeta^{M}$ is used, and $\mu_{\Omega}^{M}$ denotes the Equitable Men's Optimal Stable Matching, its outcome. Analogously, $\mu_{\Omega}^{W}$ denotes the Equitable Women's Optimal Stable Matching.

Let $P^{\Omega}$ be the preferences $P$ where we delete from men's preferences participants ranked below their mates in $\mu_{\Omega}^{W}$, and we delete from the original women's preferences the participants ranked below their mates in $\mu_{\Omega}^{M}$. The Equitable Set is the set of stable matchings in the market $\left(M, W, P^{\Omega}\right)$. Let us denote the Equitable Set by $\Omega$. 
The following example shows how the algorithm works and how the initial order of the participants gives priority to some participants over others.

EXAMPLE 1 [Roth and Sotomayor (1990, p. 70)]. Consider a market with two sets of participants $M=\left\{m_{1}, m_{2}, m_{3}, m_{4}\right\}$ and $W=\left\{w_{1}, w_{2}, w_{3}, w_{4}\right\}$ with the following preferences:

$$
\begin{aligned}
& m_{1}: w_{1}, w_{2}, w_{3}, w_{4}, m_{1}, w_{1}: m_{4}, m_{3}, m_{2}, m_{1}, w_{1}, \\
& m_{2}: w_{2}, w_{1}, w_{4}, w_{3}, m_{2}, w_{2}: m_{3}, m_{4}, m_{1}, m_{2}, w_{2}, \\
& m_{3}: w_{3}, w_{4}, w_{1}, w_{2}, m_{3}, w_{3}: m_{2}, m_{1}, m_{4}, m_{3}, w_{3}, \\
& m_{4}: w_{4}, w_{3}, w_{2}, w_{1}, m_{4}, w_{4}: m_{1}, m_{2}, m_{3}, m_{4}, w_{4},
\end{aligned}
$$

The set of stable matching of this market is represented in the following picture.

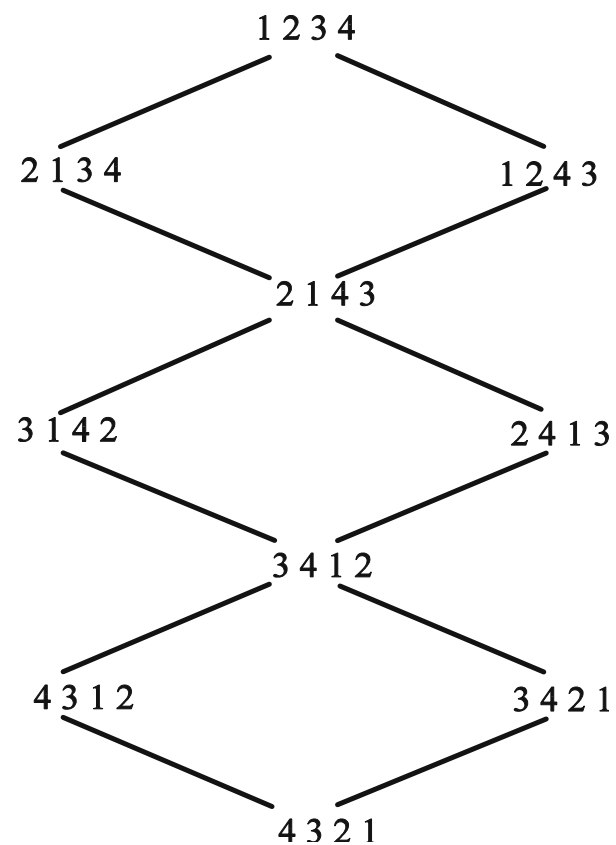

The men are omitted and matchings are represented by the number of women who are matched with men numbers one, two, three and four, respectively.

We apply $\Phi^{M}$ and $\Phi^{W}$ to achieve $P^{\Omega}$ that defines $\Omega$. The $\Phi^{M}$ goes as follows: in the first step we order the participants, all the men come first and all the women come after 
them. The $\Phi^{M}$ first step restricts participants' preferences to their first element and the participants make proposals in the selected order. There is no match in the first step. During the second step, we add the second element to the preferences of all the participants and try again in the same way. There is no match either. The outcome of the third step is the following:

\begin{tabular}{|c|c|c|c|c|c|c|c|}
\hline $\mathbf{m}_{1}$ & $\mathbf{m}_{2}$ & $\mathbf{m}_{3}$ & $\mathbf{m}_{4}$ & $\mathbf{w}_{1}$ & $\mathbf{w}_{2}$ & $\mathbf{w}_{3}$ & $\mathbf{w}_{4}$ \\
\hline $\mathrm{w}_{1}$ & $\mathrm{w}_{2}$ & $\mathrm{w}_{3}$ & $\mathrm{w}_{4}$ & $\mathrm{~m}_{4}$ & $\mathrm{~m}_{3}$ & $\mathrm{~m}_{2}$ & $\mathrm{~m}_{1}$ \\
\hline$\left[w_{2}\right]$ & {$\left[w_{1}\right]$} & {$\left[w_{4}\right]$} & {$\left[w_{3}\right]$} & $\mathrm{m}_{3}$ & $\mathrm{~m}_{4}$ & $\mathrm{~m}_{1}$ & $\mathrm{~m}_{2}$ \\
\hline $\mathrm{w}_{3}$ & $\mathrm{w}_{4}$ & $\mathrm{w}_{1}$ & $\mathrm{w}_{2}$ & {$\left[m_{2}\right]$} & {$\left[m_{1}\right]$} & {$\left[m_{4}\right]$} & {$\left[m_{3}\right]$} \\
\hline
\end{tabular}

In the above table, the first row depicts the participants while the rest of the rows depicts their preferences. In the third step, man $m_{1}$ proposes to $w_{1}$, then to $w_{2}$ and $w_{2}$ accepts his proposal. The same happens for $m_{2}$, and his proposal is accepted by $w_{1}$. After all the men propose, there is no participant unmatched and the algorithm ends. The Equitable Men's Optimal Stable Matching is

$$
\mu_{\Omega}^{M}=\left\{\left(m_{1}, w_{2}\right),\left(m_{2}, w_{1}\right),\left(m_{3}, w_{4}\right),\left(m_{4}, w_{3}\right)\right\} .
$$

The equitable women's algorithm works in the same way with all women proposing before the men. There is no match in this algorithm until the third step. It is the following:

\begin{tabular}{|c|c|c|c|c|c|c|c|}
\hline $\mathbf{w}_{1}$ & $\mathbf{w}_{2}$ & $\mathbf{w}_{3}$ & $\mathbf{w}_{4}$ & $\mathbf{m}_{1}$ & $\mathbf{m}_{2}$ & $\mathbf{m}_{3}$ & $\mathbf{m}_{4}$ \\
\hline $\mathrm{m}_{4}$ & $\mathrm{~m}_{3}$ & $\mathrm{~m}_{2}$ & $\mathrm{~m}_{1}$ & $\mathrm{w}_{1}$ & $\mathrm{w}_{2}$ & $\mathrm{w}_{3}$ & $\mathrm{w}_{4}$ \\
\hline$\left[m_{3}\right]$ & {$\left[m_{4}\right]$} & {$\left[m_{1}\right]$} & {$\left[m_{2}\right]$} & $\mathrm{w}_{2}$ & $\mathrm{w}_{1}$ & $\mathrm{w}_{4}$ & $\mathrm{w}_{3}$ \\
\hline $\mathrm{m}_{2}$ & $\mathrm{~m}_{1}$ & $\mathrm{~m}_{4}$ & $\mathrm{~m}_{3}$ & {$\left[w_{3}\right]$} & {$\left[w_{4}\right]$} & {$\left[w_{1}\right]$} & {$\left[w_{2}\right]$} \\
\hline
\end{tabular}

In the third step each woman proposes to her second element, she is accepted and the algorithm stops. The Equitable Women's Optimal Stable Matching is

$$
\mu_{\Omega}^{W}=\left\{\left(m_{1}, w_{3}\right),\left(m_{2}, w_{4}\right),\left(m_{3}, w_{1}\right),\left(m_{4}, w_{2}\right)\right\} .
$$

In this example there are two SEMs as defined in Romero-Medina (2001). This matchings are

$$
\mu_{\Omega}^{S E M 1}=\left\{\left(m_{1}, w_{3}\right),\left(m_{2}, w_{1}\right),\left(m_{3}, w_{4}\right),\left(m_{4}, w_{2}\right)\right\},
$$




$$
\mu_{\Omega}^{S E M 2}=\left\{\left(m_{1}, w_{2}\right),\left(m_{2}, w_{4}\right),\left(m_{3}, w_{1}\right),\left(m_{4}, w_{3}\right)\right\} .
$$

Both matchings are included in the Equitable Set. However, this circumstance is not general. The Equitable Set and the SEM are independent and complementary solution concepts. The SEM presents a matching that minimizes envy between men and women and it is a cardinal concept. The Equitable Set is ordinal and the matchings on the Equitable set are Rawlsian. There is another characteristic that makes the Equitable Set and the Equitable Algorithm particularly appealing. Although the Equitable Set is generically not a singleton, the Equitable Algorithm selects only one stable matching for each of the agents priority order. Therefore the solution of the Equitable Algorithm is unique for a given priority order.

\section{STRUCTURE OF THE EQUITABLE SET}

The structure of the Equitable Set $\Omega$ gives us a reference that can be used to select a particular matching in the set. Let us introduce some concepts in order to prove that the Equitable Set is a sublattice of the set of stable outcomes in $(M, W, P)$.

The set of stable matchings is a lattice under the preference relation of the participants in each side of the market. Recall that a partially ordered set is a lattice $(L ; \vee, \wedge)$ if sup and inf exists for all $a, b \in L$. We formally define the concepts of Sublattice and rotation. Both concepts are used in this section.

DEFINITION 1: A Sublattice $\mathcal{S}=(K ; \vee, \wedge)$ of the lattice $\Upsilon=$ $(L ; \vee, \wedge)$ is defined on a nonvoid subset $K \subseteq L$ with the property that $a, b \in K$ implies that $a \wedge b, a \vee b \in K(\vee, \wedge$ taken in $\Upsilon)$, and the $\wedge$ and the $\vee$ of $\mathcal{S}$ are restrictions to $K$ of the $\wedge$ and the $\vee$ of $\Upsilon$.

We shall now formally define the concept of rotation. Let $\mu$ be a stable matching. For any man $m_{i}$ let $s_{\mu}\left(m_{i}\right)$ denote the first woman $w_{j}$ on $m_{i}$ 's list such that $w_{j}$ strictly prefers $m_{i}$ to $\mu\left(w_{j}\right)$ (her partner in $\mu$ ). Let $\operatorname{ext}_{\mu}\left(m_{i}\right)$ denote the partner in $\mu$ of a woman $s_{\mu}\left(m_{i}\right)$. 
DEFINITION 2: Let $\rho=\left(m_{\mathbf{0}}, w_{\mathbf{0}}\right),\left(m_{\mathbf{1}}, w_{\mathbf{1}}\right), \ldots,\left(m_{\mathrm{r}-\mathbf{1}}, w_{\mathrm{r}-\mathbf{1}}\right)$ be an ordered list of pairs in a stable matching $\mu$ such that for each $i(0 \leqslant i \leqslant r-1) m_{i+1}$ is next ${ }_{\mu}\left(m_{i}\right)$, where $i+1$ is taken modulo $r$, i.e., $m_{r}=m_{0}$ and $w_{r}=w_{0}$. Then $\rho$ is called an $m$ rotation (exposed) in $\mu$, and we say that $m_{i}$ (or $w_{j}$ ) is in a $m$ rotation $\rho$ if there is a pair $\left(m_{i}, w_{j}\right)$ in the ordered list defining $\rho$.

DEFINITION 3: If $\mu$ is a stable matching, and $\rho=\left\{\left(m_{\mathbf{0}}, w_{\mathbf{0}}\right)\right.$, $\left.\left(m_{1}, w_{\mathbf{1}}\right), \ldots,\left(m_{\mathbf{r}-\mathbf{1}}, w_{\mathbf{r}-1}\right)\right\}$ is an $m$-rotation exposed in $\mu$, then $\mu / \rho$ is defined to be a matching in which each man not in $\rho$ stays married to his partner in $\mu$, and each man $m_{i}$ in $\rho$ marries $w_{i+1}=s_{\mu}\left(m_{i}\right)$. The transformation from $\mu$ to $\mu / \rho$ is called elimination of $\rho$ from $\mu$.

DEFINITION 4: Let $\rho=\left\{\left(m_{\mathbf{0}}, w_{\mathbf{0}}\right),\left(m_{\mathbf{1}}, w_{\mathbf{1}}\right), \ldots,\left(m_{\mathbf{r}-\mathbf{1}}, w_{\mathbf{r}-1}\right)\right\}$ be an $m$-rotation. We say that $\rho$ moves $m_{i}$ down from $w_{i}$ to $w_{i+1}$, and moves $w_{i}$ up from $m_{i}$ to $m_{i-1}$. If $w$ is either $w_{i}$ or is strictly between $w_{i}$ and $w_{i+1}$ in $m_{i}$ 's list, then $\rho$ moves $m_{i}$ below $w$. Similarly, $\rho$ moves $w_{i}$ above $m$ if $m$ is $m_{i}$, or is strictly between $m_{i}$ and $m_{i-1}$ in $w_{i}$ 's list.

Definition 4 describes what happens to each person in $\rho$ when $\rho$ is eliminated from a matching $\mu$ in which it is exposed. Essentially, each man in the rotation will have as his match the next acceptable women in a stable matching.

For any man $m_{i}$ and for any woman $w_{j}$ in $(M, W, P)$ there is at most one $m$-rotation that moves $m_{i}$ down to $w_{j}$, and $w_{j}$ up to $m_{i}$. A $m$-rotation may be exposed in more than one matching. It is not necessarily associated with a unique matching.

Let $\Pi^{m}(\Gamma(P))$ be the set of $m$-rotations between the set of stable matchings $\Gamma(P)$. There is another set of $w$-rotations $\Pi^{w}(\Gamma(P))$. Let us denote by $\Pi(\Gamma(P))$ the set $\Pi^{m}(\Gamma(P)) \cup$ $\Pi^{w}(\Gamma(P))$ of both $m$ and $w$ rotations.

THEOREM 2. The Equitable Set with the preferences $P$ is a sublattice of $\Gamma(P)$. 
In order to prove Theorem 2 we have to establish some results.

DEFINITION 5: The transformation from the preferences $P$ to $P^{\prime}$ is regular if and only if:

(a) $\Gamma\left(P^{\prime}\right) \subseteq \Gamma(P)$ and

(b) $\Pi\left(\Gamma\left(P^{\prime}\right)\right) \subseteq \Pi(\Gamma(P)) \cup \emptyset$.

In other words, a transformation $P^{\prime}$ of $P$ is regular if and only if comparing the markets $(M, W, P)$ and $\left(M, W, P^{\prime}\right)$, the rotations are exposed and the set of stable matchings in $\left(M, W, P^{\prime}\right)$ are a subset of the rotations and the stable matchings set in the original market.

LEMMA 3. Every regular transformation $P^{\prime}$ of $P$ generates a new set of stable matchings $\Gamma\left(P^{\prime}\right)$ which is a sublattice of $\Gamma(P)$.

Proof. We have two cases:

(a) $P^{\prime}=P$ which is straightforward.

(b) $P^{\prime} \neq P$. As $\Gamma\left(P^{\prime}\right)$ is a lattice it means that for all $\mu^{a}, \mu^{b} \in$ $\Gamma\left(P^{\prime}\right) ; \mu^{a} \wedge \mu^{b} \in \Gamma\left(P^{\prime}\right)$ and $\mu^{a} \vee \mu^{b} \in \Gamma\left(P^{\prime}\right)$. Because $P^{\prime}$ is a regular transformation of $P$ then $\mu^{a}, \mu^{b} \in \Gamma(P)$.

Let $\mu^{a} \wedge \mu^{b}=\mu^{\beta}$ and $\mu^{a} \vee \mu^{b}=\mu^{\alpha}$. There is an $m$-rotation or a set of $m$-rotations $\pi_{\alpha}, \pi_{\beta}, \varpi_{\alpha}, \varpi_{\beta} \in \Pi\left(\Gamma\left(P^{\prime}\right)\right)$ such that

$$
\begin{aligned}
\mu^{\alpha} / \pi_{\alpha} & =\mu^{a}, \\
\mu^{\alpha} / \pi_{\beta} & =\mu^{b}, \\
\mu^{a} / \varpi_{\alpha} & =\mu^{\beta}, \\
\mu^{b} / \varpi_{\beta} & =\mu^{\beta} .
\end{aligned}
$$

We need to check that $\mu^{a} \wedge \mu^{b}=\mu^{\beta}$ and $\mu^{a} \vee \mu^{b}=\mu^{\alpha}$ under $P$ in $\Gamma(P)$.

First, $\mu^{\alpha}$ and $\mu^{\beta}$ belong to $\Gamma(P)$ because $\Pi\left(\Gamma\left(P^{\prime}\right)\right) \subseteq$ $\Pi(\Gamma(P)) \cup \emptyset$. Moreover, all the stable matchings between $\mu^{a}$ and $\mu^{b}$ and their infimum and supremum in $\Gamma\left(P^{\prime}\right)$ belong to $\Gamma(P)$. Suppose that a stable matching $\mu^{c}$ in $\Gamma(P)$ exists such that $\mu^{a} \vee \mu^{b}=\mu^{c}$ in $\Gamma(P)$ and $\mu^{c} \notin \Gamma\left(P^{\prime}\right)$. This means that there is a set of rotations $\omega$ such that $\mu^{c} / \omega=\mu^{a}$ and $\omega \nsubseteq \Pi\left(\Gamma\left(P^{\prime}\right)\right)$. Then, there is at least one rotation $\rho^{*} \in \omega$ such 
that $\rho^{*} \notin \Pi\left(\Gamma\left(P^{\prime}\right)\right)$. We know that between the men's optimal stable matching and each one of the elements in the set of stable matchings we always find the same rotations ${ }^{1}$. It implies that if $\rho^{*} \notin \Pi\left(\Gamma\left(P^{\prime}\right)\right)$, it must be eliminated before $\mu^{\alpha}$. Therefore, there is no possibility for $\mu^{c}$ to be the supremum of $\mu^{a}$ and $\mu^{b}$ under $P$ in $\Gamma(P)$.

Analogously, we can prove that $\mu^{\beta}$ must be the infimum of $\mu^{a}$ and $\mu^{b}$ under $P$ in $\Gamma(P)$.

LEMMA 4. $\mu_{\Omega}^{M}$ and $\mu_{\Omega}^{W}$ are the extreme points of $\Gamma\left(P^{\Omega}\right)$.

This result is a direct consequence of the definition of $P^{\Omega}$.

LEMMA 5. $P^{\Omega}$ is a regular transformation of $P$.

Proof. We check the two conditions:

(a) $\Gamma\left(P^{\prime}\right) \subseteq \Gamma(P)$ and

(b) $\Pi\left(\Gamma\left(P^{\prime}\right)\right) \subseteq \Pi(\Gamma(P)) \cup \emptyset$.

By Proposition 1, and the definition of $P^{\Omega}$, we already know that $\mu_{\Omega}^{W}$ and $\mu_{\Omega}^{M}$ belong to $\Gamma(P)$ and $\Gamma\left(P^{\Omega}\right)$. By the definition of $P^{\Omega}$ we also know that all the elements of participants' preferences between $\mu_{\Omega}^{W}$ and $\mu_{\Omega}^{M}$ remain in their positions in $P$. This means that all the rotations in $\Pi\left(\Gamma\left(P^{\Omega}\right)\right)$ are in $\Pi(\Gamma(P))$. Since these rotations are equal to all the stable matching in $\Gamma\left(P^{\Omega}\right)$, they also belong to $\Gamma(P)$.

Proof of Theorem 2. By Lemmas 3-5 we know that the Equitable Set is a sublattice of the lattice of stable matchings in every matching market.

The fact that the Equitable Set is a sublattice from the original set of stable matching guarantees that the matching on the Equitable Set are connected by rotations in the original market. It also guarantees that the rotations on the Equitable Set are a subset from the rotations on the original market. Therefore, no distortion is introduced on the way stable matchings are related. 


\subsection{Computing the equitable set}

The structure of the Equitable Set allows the Equitable Algorithm to select one particular matching from the stable set by choosing one particular order of agents. This order generates a priority between agents that will favour those that act first in the Equitable Algorithm. Thus, we no longer have to choose between men or women. The Equitable Algorithm can prioritize some men and some women, and find a compromise matching that is stable and favours then with respect to the rest of the agents.

The following result establishes how to achieve any matching in $\Omega$. It also shows how no allocation outside the Equitable Set can be generated by the Stable Algorithm.

THEOREM 6. For any stable matching $\mu_{\Omega}^{\circ} \in \Omega$ there is always at least one initial order of the participants $\zeta^{\circ}$ such that $\Phi^{\circ}$ produces $\mu_{\Omega}^{\circ}$.

Proof. If we have only one stable matching, every order of the participants selects this particular matching (by Proposition 1) and the result becomes trivial. Assume that there is more that one element in $\Omega$. We already know that $\Phi^{M}$ produces $\mu_{\Omega}^{M}$. If $\Omega$ has more than one element, it means that there is at least one rotation exposed in $\mu_{\Omega}^{M}$ and this rotation, denoted by $\rho^{1}$, can be eliminated generating a new stable matching in $\Omega$. Call this new stable matching $\mu_{\Omega}^{M} / \rho^{1}=\mu_{\Omega}^{1}$. The center of the argument is to show how a change in $\zeta^{M}$ can generate $\mu_{\Omega}^{1}$ through $\Phi$.

Define a new original position for the participants $\zeta^{0}$, where $\zeta^{0}=\zeta^{M}$ and let $\zeta^{1}$ be identical to $\zeta^{0}$, except for the agents involved in $\rho^{1}$. The women in $\rho^{1}$ are situated in $\zeta^{1}$ just before the men who are their partners in $\mu_{\Omega}^{M}$.

First, we shall prove that the change from $\zeta^{0}$ to $\zeta^{1}$ cannot affect the mates of the participants not in $\rho^{1}$. The proof proceeds by contradiction, assuming that $\zeta^{1}$ generates a marching $\mu_{\Omega}^{\prime}$ where $w_{1} \in \rho^{1}$ is matched with $m_{i} \notin \rho^{1}$. In that case, $\mu_{\Omega}^{M} P_{m^{\prime}} \mu_{\Omega}^{\prime}$, because the set $\Omega$ is a lattice. The mate of $m_{i}$ in

$\mu_{\Omega}^{M}$ is $w_{i}$. Therefore $m_{i}$ prefers $w_{i}$ to $w_{1}$. Even if $m_{i}$ has 
been forced to accept $w_{1}$ 's proposal when $w_{i}$ was available, $w_{i}$ always has a chance to propose to $m_{i}$ later; if she does not, it must be because $w_{i}$ has a partner better than $m_{i}$. The same happens with the mate of $w_{i}$ 's new partner in $\mu_{\Omega}^{M}$. Therefore there is a rotation $\rho=\left\{\left(m_{0}, w_{0}\right),\left(m_{1}, w_{1}\right), \ldots,\left(m_{r-1}, w_{r-1}\right)\right\}$ exposed in $\mu_{\Omega}^{M}$ such that $\left\{\left(m_{i}, w_{i}\right),\left(m_{1}, w_{1}\right)\right\} \in \rho$. However, no element can be in two rotations exposed in the same stable matching. It means that $\rho=\rho^{1}$, a contradiction.

We proceed to show how the order $\zeta^{1}$ forces the rotation $\rho^{1}$ to be eliminated from the matching $\mu_{\Omega}^{M}$. Call $w_{1}$ the first woman to make a proposal in $\zeta^{1}$. By the definition of rotation, when $m_{1}$ is able to make her a proposal, her mate in $\mu_{\Omega}^{M} / \rho^{1}=m_{2}$ is already available. $w_{1}$ prefers $m_{2}$ to $m_{1}$, then she sends $m_{2}$ a proposal and he has to accept. The same thing happens with all women in $\rho^{1}$ who are now proposing before their mates in $\mu_{\Omega}^{M}$ propose. It leads to the elimination of the rotation $\rho^{1}$, which is what we wanted to prove.

Any rotation can be eliminated using the same procedure from a given matching and the order of agents that generates this matching. Starting in $\mu_{\Omega}^{*}$, we only have to place each woman in the rotation to eliminate $\rho^{i}$ before the men that correspond to her in that rotation. This way we transform $\zeta^{*}$ into $\zeta^{i}$. As we have shown before the order $\zeta^{i}$ in the initial step of the procedure $\Phi$ will generate the matching $\mu_{\Omega}^{*} / \zeta^{i}$. Given that there is a path of rotations that leads from $\mu_{\Omega}^{M}$ to any matching in $\Omega$, it means that there is a starting order $\zeta^{\prime}$ that eventually will lead algorithm $\Phi$ to the matching $\mu_{\Omega}^{\prime}$.

PROPOSITION 7. The algorithm $\Phi^{M}$ generates the matching $\mu_{\Omega}^{M}$ regardless of the initial order of men in $\zeta^{M}$.

When all men precede all women in the initial order $\zeta^{M}$, the Equitable Procedure generates the stable matching $\mu_{\Omega}^{M}$. This matching is unique. This results follows from Theorem 6 and the fact that any change in men's order will not generate a rotation elimination (see Definition 3). Therefore, the 
matching obtained by the algorithm will not change. An analogous result can be established for $\zeta^{W}$.

As stated above, by fixing an order of the participants we are determining the final matching selected by the Equitable algorithm in $\Omega$. If we want to benefit a set of participants we only need to situate them at the beginning of a particular order $\zeta$ and $\Phi$ gives us the stable matching in $\Omega$ that treats them better than those that are situated in $\zeta$ after them. It is clear now how the order where all the women are situated before any man $\zeta^{W}$ generates $\mu_{\Omega}^{W}$. Therefore, both $\mu_{\Omega}^{W}$ and $\mu_{\Omega}^{M}$ are unique and independent of the order that men or women have among themselves. This is because no change in the relative order of men or women can eliminate a rotation when either all women precede all men or vice versa.

In Example 1, we can see that there are two non-extreme stable matchings on $\Omega$. Those stable matchings can be achieved by $\Phi$ if we specify the right order of the participants. In this case, the order to achieve matching $\mu^{5}$ is $w_{3}, w_{2}, m_{2}, m_{3}, \ldots$ To achieve $\mu^{6}$ the adequate order is $w_{4}, w_{1}, m_{1}, m_{4}, \ldots$ Notice that the other participants' positions are not relevant because they have no chance to make proposals at the third step.

\section{RAWLSIAN MATCHINGS}

The Equitable Set has the property of reducing the conflict between agents to the minimum that is compatible with stability. This is because no agent is forced to accept a mate if there is a stable matching where the agent with the worse possible mate can be better of. Moreover, this minimum is not general for all the market but effective for each submarket of related agents. This approach is in the same spirit as the original non-mathematical treatment of Rawls (1971) later used in Masarani and Gokturk $(1986,1989)$.

Given a matching market $(M, W, P)$ and its related set of stable matchings $\Gamma(P)$, let $\Gamma(\sigma)$ for $\sigma \in M \cup W$ be the set of elements that may be assigned to the element $\sigma$ in a stable matching on the market $(M, W, P)$. We can reduce the pref- 
erence list of all individuals $P$ to its $\Gamma(\sigma)$ elements. Let us denote these preferences by $P^{\Gamma}$.

DEFINITION 6: Given a marriage market $\left(M, W, P^{\Gamma}\right)$ a minimal decomposition or a decomposition is a set of men $T \subseteq M$, a set of women $Y \subseteq W$, and a set of preference list, where the men in $T$ rank only women in $Y$ and the women in $Y$ rank only men in $T$.

According to our definition, a decomposition is a partition of the participants in two or more $(T, Y)$ submarkets, $\left\{\left(T_{i}, Y_{i}\right), i=1, \ldots, k\right\}$, so that the set $\Gamma(P)$ is the Cartesian product of the sets $\Gamma\left(T_{i}, Y_{i}\right), i=1, \ldots, k$. That is, if we arbitrarily take one stable matching from each of the $k$ submarkets of the decomposition, then the union of these matchings is a stable matching in $\Gamma(P)$, and every stable matching in $\Gamma(P)$ can be obtained in this way ${ }^{2}$.

Let us denote the position of the woman $w_{j}$ in $m_{i}$ 's list as $r_{m_{i}}\left(w_{j}\right)$. Let $r_{m_{i}}(\mu)$ be the position of the mate of $m_{i}$ in the matching $\mu$ in $m_{i}$ 's list.

DEFINITION 7: A matching $\mu$ dominates in the sense of Rawls another matching $\mu^{\prime}$ if and only if

$$
\max _{\text {for all }\left(m_{i}, w_{j}\right) \in \mu}\left[r_{w_{j}}(\mu), r_{m_{i}}(\mu)\right]<\max _{\text {for all }\left(m_{i}, w_{j}\right) \in \mu^{\prime}}\left[r_{w_{j}}(\mu), r_{m_{i}}(\mu)\right] .
$$

DEFINITION 8: A stable matching $\mu \in \Gamma(P)$ is Rawlsian by Partitions if and only if the stable matching $\mu$ is not dominated in the sense of Rawls by any other stable matching in this partition.

PROPOSITION 8: All the matchings in $\Omega$ are Rawlsian by partitions.

Proof. Suppose that they are not. Then there exists a stable matching such that $\mu_{\Omega}$ is not Rawlsian by partitions. It means that there exists a $\mu_{\Omega}^{\prime}$ such that

$$
\begin{aligned}
& \left.\max _{\text {for all }} \quad m_{i}, w_{j}\right) \in\left(T_{i}, Y_{i}\right) \text { in } \mu_{\Omega}^{\prime} \\
& \quad \max _{\text {for all }\left(m_{i}, w_{j}\right) \in\left(T_{i}, Y_{i}\right)} \text { in } \mu_{\Omega}\left[r_{w_{j}}(\mu), r_{m_{i}}(\mu)\right] \\
& {\left[r_{w_{j}}(\mu), r_{m_{i}}(\mu)\right] .}
\end{aligned}
$$


However, the partner of the worse participant in $\left(T_{i}, Y_{i}\right)$ in the matching $\mu_{\Omega}$, let's say $m_{z}$ is forced by the algorithm to propose to his partner in $\mu_{\Omega}^{\prime}$, as are the other men in $\left(T_{i}, Y_{i}\right)$. However at the same stage where $w_{z}$ is not available for $m_{z}$ there is a potential partner for her, her mate in $\mu_{\Omega}^{\prime}$. If all the participants in $\left(T_{i}, Y_{i}\right)$ find a mate at stage $\mathrm{K}$ by $\Phi$, none of them can make new proposals. By the definition of partition, no participant $\sigma \notin\left(T_{i}, Y_{i}\right)$ may affect the outcome. It means that $\mu_{\Omega} \notin \Omega$. A contradiction.

\section{FINAL REMARKS}

The Equitable Algorithm has interesting characteristics. It allows the selection of fair matchings in the sense that there is not a stable matching where the agent with the worse possible mate can be better of. It also allows to discriminate in the process of allocation in favor of some agents. The Equitable Set is the set of stable matchings that can be selected by the Equitable Algorithm.

A natural extension of the paper is to select $\zeta$ in a random way. In that case, the matching finally achieved by the mechanism will be decided by chance. In this way we can guarantee that any matching on the Equitable Set can be selected as final matching and men and women have equal chances, ex ante, to benefit from the algorithm. Of course, all the properties of the Equitable Set will be satisfied.

This procedure is not related to Roth and Vande (1990, 1991). They studied a randomized matching mechanism that considers starting from any matching and then randomly choosing a blocking pair and satisfying it if such a pair existed. They showed that for any starting matching it is possible to find a finite sequence of matchings such that it reaches a stable matching. The key issue is that, with this procedure, Roth and Vande Vate can obtain any stable matching. If $\zeta$ is selected at random and the algorithm $\Phi$ is applied, this 
is not the case. In that situation, only matchings on the Equitable Set can be achieved.

\section{ACKNOWLEDGEMENTS}

I thank Professor Salvador Barberá for his very useful suggestions. I am grateful to José Alcalde, David Perez-Castrillo, Jordi Massó, Carmen Herrero, Amparo Urbano, Jorge Nieto and Amed Alkan for their comments. All errors are my own responsibility. Financial support from Ministerio de Ciencia y Tecnologia under project BCE-2002-02194 and Comunidad de Madrid 06/HSE/0170/2004 is gratefully acknowleged.

\section{NOTES}

1. Gusfield and Irving (1989) Lemma 2.5.7 and Corollary 2.5.2.

2. Gusfield and Irving (1989) Theorems 3.4.4 and 3.4.5.

\section{REFERENCES}

Bennett, E. (1988), Consistent bargaining conjectures in marriage and matching, Journal of Economic Theory 45, 392-407.

Gusfield, D. and Irving, R.W. (1989), The Stable Marriage Problem, Structure and Algorithms, Cambridge, MA: M.I.T. Press.

Knuth, D. (1976), Marriages Stables, Les Presses de l'Université de Montréal.

Masarani, F. and Gokturk, S.S. (1986), A pareto optimal characterization of rawls' Social Choice Mechanism, Journal of Mathematical Economics $15,157-170$.

Masarani, F. and Gokturk, S.S. (1989), On the existence of fair matching algorithms, Theory and Decision 26, 305-322.

Rawls, J. (1971), A Theory of Justice, Cambridge, MA: Harvard University Press.

Rochford, S.C. (1984), Symmetrically pairwise-bargained allocations in an assignment market, Journal of Economic Theory 34, 262-281.

Romero-Medina, A. (2001), Sex-equal stable matching, Theory and Decision 50, 197-212.

Roth, Alvin E. and Sotomayor, M. (1988), Interior points in the core of two-sided matching markets, Journal of Economic Theory 45, 85-101. 
Roth, Alvin E. and Sotomayor, M. (1990), Two-Sided Matchings Markets: A Study in Game Theoretic Modeling and Analysis, Econometrics Society Monograph, Cambridge, MA: Cambridge University Press.

Roth Alvin E. and Vande Vate, J.H. (1990), Random paths to stability in two-sided matching, Econometrica 58, 1475-1480.

Roth, Alvin E. and Vande Vate, J.H. (1991), Incentives in two-sided matching with random mechanism, Economic Theory 1, 33-44.

Address for correspondence: Antonio Romero-Medina, Departamento de Economia, Universidad Carlos III de Madrid, C/ Madrid 126, 28903, Getafe, Madrid, Spain. E-mail: aromero@eco.uc3m.es 